\title{
The Otoconia of the Vertebrate Gravity Receptor Organs: Biomineral Structure and Interactions with the Protein Filament Matrix
}

\author{
Ulysses Lins $^{1,2}$, Marcos Farina ${ }^{3}$, Ruediger Thalmann ${ }^{4}$, and Bechara Kachar ${ }^{1}$ \\ ${ }^{1}$ Section on Structural Cell Biology, NIDCD, NIH, Bethesda, MD 20892-4163, USA; \\ ${ }^{2}$ Instituto de Microbiologia, Universidade Federal do Rio de Janeiro, RJ, 21941-590, Brazil. \\ ${ }^{3}$ Departamento de Anatomia, Universidade Federal do Rio de Janeiro, RJ, 21941-590, Brazil. \\ ${ }^{4}$ Department of Otolaryngology, Washington University School of Medicine, St. Louis, MO 63110.
}

A unique feature of the saccule and utricle, is the mass of otoconia, overlaying a gelatinous matrix on the surface of the sensory epithelium. Otoconia are composites of organic matter and one of the three crystal forms of calcium carbonate: calcite, vaterite and aragonite. Each crystal form has characteristic crystallographic and physical properties. In mammals, otoconia contain proteins and calcium carbonate in the form of composite calcite crystals [1]. In amphibians, calcite otoconia are present in the utricle whereas both pinacoid and prismatic shaped aragonitic otoconia are found in the saccule. Fish usually contains aragonitic otoconia, with some reported exceptions that contain calcite, vaterite and calcium carbonate monohydrate [2]. We used a combination of imaging techniques including quick-freeze, deep-etching to examine the otoconial mass of the frog saculus and the guinea pig utricle. The deep-etching step exposed large expanses of intact and fractured otoconia, showing the fine structure and relationship between their internal crystal structure, their surface components and the filament matrix in which they are embedded. The guinea pig otoconia have a unique compact central core meshwork of filaments and a composite outer shell of ordered crystallites and macromolecular aggregates. The core is formed by a tight meshwork of filaments $14 \mathrm{~nm}$ in diameter with a mesh size of $32 \mathrm{~nm}$, giving the central core a characteristic dense texture. A distinct network of $20 \mathrm{~nm}$ beaded filaments covers the surface of the otoconia. The otoconia are interconnected and secured to the gelatinous matrix by surface adhesion and by confinement within a loose inter-otoconial filament matrix. The gelatinous matrix is a dense network made of yet another type of filaments, $22 \mathrm{~nm}$ in diameter that are cross-linked by shorter filaments, characteristically $11 \mathrm{~nm}$ in diameter. Recently, two novel proteins called otoconin-22 and otoconin90 were identified and characterized as the major otoconial matrix protein in frog and mouse respectively [3]. We used an antibody specific to the N-terminal sequence of otoconin-90 and postembedding immunogold labeling to study the distribution of otoconin-90 in mouse otoconia. We found otoconin-90 highly expressed in the central core region as well as in the outer shell regions of the otoconia indicating that it is the main protein of the core filament meshwork as well as a key component of the composite crystalline matrix. The presence of otoconin-90 between the calcium carbonate crystallites in the periphery of the otoconia clearly aligned to the surface of the structure is important because it suggests that this protein, possibly in association with other otoconins, may direct the assembly and orientation of crystallites and prevent excessive growth of the otoconia by inhibiting additional precipitation of calcium carbonate. Our freeze-etching and immunolocalization data provide a structural framework to consider the molecular, mechanical and developmental nature of the components of the otoconial complex.

1. S. Mann et al., Proc. R. Soc. Lond. B Biol. Sci. 218 (1983) 415.

2. A. Oliveira et al., Naturwissenschaften 83 (1996) 133.

3. Y. Wang et al., Proc. Natl. Acad. Sci. USA 95 (1998) 15345. 

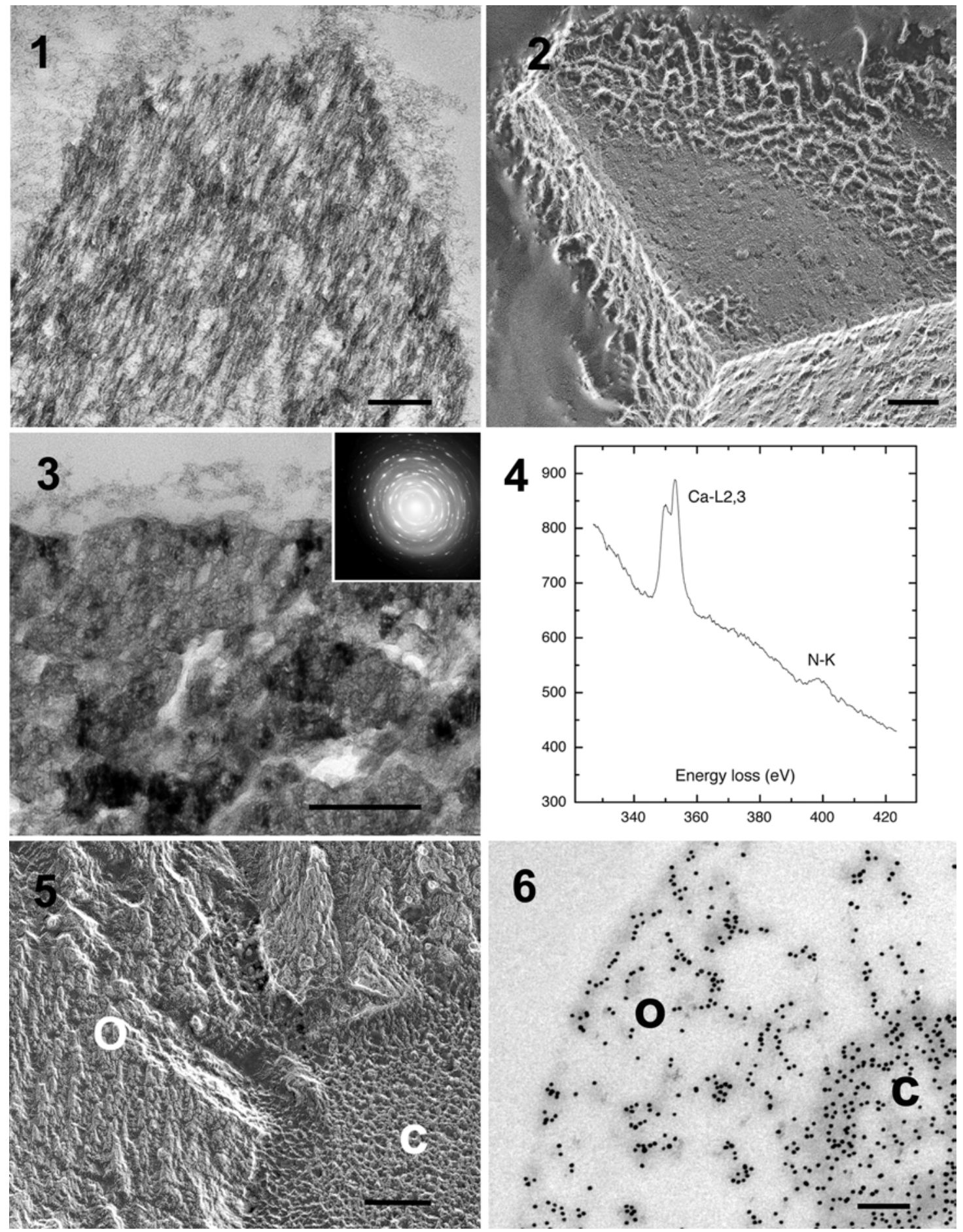

Figures 1) Thin section TEM of mouse otoconia showing numerous fibrils; 2) Freeze-etching of guinea pig otoconium showing its exposed surface components and the filament matrix in which it is embedded; 3) High magnification TEM of mouse otoconia showing its composite structure, inset shows electron diffraction pattern typical of calcite; 4) EELS of mouse otoconium showing the presence of calcium from the carbonate and nitrogen from organic matter. 5) Deep-etching of otoconium showing both the central core (c) and outer layer (o); 6) Immunogold labeling showing a pattern of distribution of otoconin-90 in the outer layer (o) and core (c). Bar equals $0.2 \mu \mathrm{m}$ 